\title{
Incidence and clinical predictors of infections in patients treated with severe systemic ANCA-associated vasculitis
}

\author{
Á. HARIS ${ }^{1 *}$ (]) K. POLNER ${ }^{2}$, J. ARÁNYI ${ }^{2}$, H. BRAUNITZER ${ }^{2}$ and I. KASZÁS ${ }^{3}$ \\ ${ }^{1}$ 1st Department of Internal Medicine and Nephrology, Péterfy Hospital and Outpatient Clinic, \\ Budapest, Hungary \\ ${ }^{2}$ Nephrology Department, Szent Margit Hospital, Budapest, Hungary \\ ${ }^{3}$ Pathology Department, Szent Margit Hospital, Budapest, Hungary
}

Received: July 27, 2020 - Accepted: September 23, 2020

Published online: March 25, 2021

(c) 2021 The Author(s)

\begin{abstract}
Background: Immunosuppressive therapy has improved the outcome of ANCA-associated vasculitis (AAV), but infectious morbidity and mortality remained high. Recognizing its risk factors seems crucial for prevention, aiming to increase survival of these patients. Methods: We investigated the incidence and types of infections and assessed predictive factors in 132 patients with severe systemic AAV. Results: Patients with lower than median incidence of total infections/patient-year during induction had lower baseline serum creatinine, dialysis requirement and Charlson comorbidity index (CCI), compared to those with higher than median incidence $(P=0.037 ; P=0.024 ; P=0.001$; respectively). In subgroups with below and above than median number of severe infections/patient-year during induction, differences were found in baseline creatinine $(P=0.002)$ and dialysis requirement $(P=0.001)$; comparing the same cohorts during maintenance immunosuppression, baseline dialysis requirement, diabetes, CCI, and dose of cyclophosphamide (CYC) administered as induction therapy differed significantly $(P=0.019 ; P=0.015 ; P=0.001 ; P=0.015$, respectively). Severe infections were predicted by baseline serum creatinine (OR 1.002 [CI 1.001-1.003]) and pulmonary manifestation (OR 2.153 [CI 1.017-4.560]) during induction immunosuppression. In multivariable Cox regression model all-cause mortality was independently predicted by severe infection (HR 1.998 [CI 1.214-3.287]). Among the 168 positive cultures Gram-negative bacteria were responsible for blood stream infections in $33 \%$, and respiratory tract infections in $72 \%$. Conclusions: Advanced renal failure, pulmonary involvement and high degree of comorbidities increase the risk of infection in AAV. Those who suffer
\end{abstract}

\footnotetext{
${ }^{*}$ Corresponding author. 1st Department of Internal Medicine and Nephrology, 8-20 Péterfy street, 1076 Budapest, Hungary. Tel.: +361461 4798. E-mail: agnesharis@hotmail.com
} 
infection during induction immunosuppression have worse long-term survival. Our findings indicate the need for high vigilance for infections and close follow-up of comorbidities when treating AAV.

\section{KEYWORDS}

ANCA-associated vasculitis, comorbidity, immunosuppression, infections, mortality, survival

\section{INTRODUCTION}

ANCA-associated vasculitis (AAV) is a systemic autoimmune disease which affects several organs. Beside non-specific constitutional signs, it most frequently damages the kidneys, and if left untreated, causes severe renal failure. More than $50 \%$ of patients develop pulmonary involvement, which may present as life-threatening pulmonary hemorrhage. Other organs may also be involved in the small-vessel necrotizing pathology. All of these manifestations cause substantial morbidity and mortality [1-5].

Immunosuppressive therapy has significantly improved the outcome of the disease. Before the 1970s the first-year mortality of patients with systemic AAV was higher than $85 \%$, but corticosteroid and cytotoxic agents, recently rituximab administration resulted in favorable short- and long-term survival $[4,6]$. Yet, treatment-associated complications develop frequently, and infectious morbidity of AAV remained high even in the modern era of immunosuppression (ISU) [7-9]. Infections represent the most important cause of mortality as well [10]. Understanding the risk factors associated with infection development in AAV seems crucial for prevention, aiming to increase quality of life and survival of these patients.

The aims of this single center retrospective cohort study were to assess the incidence and types of infections, document positive microbiological cultures, and determine predictive factors of this complication in patients with severe systemic AAV. As the intensity of ISU differs substantially during induction and maintenance ISU, we evaluated infectious complications separately in these periods.

\section{MATERIALS AND METHODS}

All consecutive patients, diagnosed between 1999 and 2019 with AAV in St. Margit Hospital Nephrology Center were considered for this study. Last follow-up was the date of death or end of study, September 30, 2019.

Diagnosis of necrotizing small vessel vasculitis was defined by clinical presentation compatible with AAV, positive ANCA serology and/or kidney biopsy, according to the definitions of 2012 Chapel Hill Consensus Conference [11]. Renal manifestation was confirmed by microscopic hematuria, active urinary sediment and pauci-immune necrotizing glomerulonephritis proved by specimen of kidney biopsy, obtained in all but 11 patients in whom it was omitted because of life threatening condition or refusal by the patient. All these patients were ANCA positive. We defined our patients' condition as having severe disease, as all of them had rapidly progressive glomerulonephritis. Exclusion criteria included combined ANCA and antiglomerular basement membrane positivity. Of the 132 participants, 16 were diagnosed with 
renal limited AAV, 50 patients had granulomatosis with polyangiitis, 64 had microscopic polyangiitis and 2 persons had eosinophilic granulomatosis with polyangiitis.

Induction ISU treatment protocol included intravenous (IV) boluses of methylprednisolone (MP), daily 500-1,000 mg for three consecutive days, followed by $1 \mathrm{mg} / \mathrm{kg}$ per os (po) for one month, which was gradually decreased over 5-6 months to the maintenance of $4 \mathrm{mg}$ given daily or every other day. Cyclophosphamide (CYC) in a dose of $9-11 \mathrm{mg} / \mathrm{kg}$ depending on age and renal function was administered in monthly IV boluses six times, than repeated at months 9 and 12 after diagnosis. This standardized therapy was followed by $91 \%$ of participants. When we analyzed their data separately, the results were comparable to the findings of the whole cohort. Five plasmapheresis (PLEX) treatments were performed for all but 14 patients, in whom it was omitted due to technical reasons or refusal by the patients. After 12 months, low dose MP and, at the discretion of the attending nephrologist, azathioprine were introduced as long-term maintenance therapy. In case of relapse, induction ISU and PLEX sessions were repeated, except for one patient who received rituximab instead of CYC. This treatment protocol was an approved therapy in Hungary not only in the early period of this study but also in the recent years due to limited accessibility of rituximab.

Remission was defined by disappearance of clinical disease activity, stabilization or improvement of kidney function and decrease of Birmingham Vasculitis Activity Score (BVAS) to zero, and relapse, as recurrence of the presenting symptoms or appearance of a new organ involvement attributable to AAV, characterized by increasing BVAS which had previously reached 0 , and requiring re-induction of ISU. We defined periods of induction ISU as treatment of active disease in the first 6 months following diagnosis or relapse, and maintenance ISU as therapy administered for maintaining remission.

The total number of infections requiring antibiotic treatment was documented, and the incidence of infections/patient-year (infections/pt-yr) during induction (6 months following diagnosis and also when treating severe relapse) and maintenance ISU was calculated. Infections were categorized as severe (sepsis, endocarditis, meningitis, pneumonia, abscess, pyelonephritis, CAPD-related peritonitis), moderate (upper respiratory tract-, joint-, enteral infections, cellulitis, zoster) and mild (lower urinary tract infections and soor). Pneumonia was differentiated from pulmonary manifestations of AAV by clinical characteristics and findings on chest XR, occasionally on CT scan or bronchoscopy; urinary tract infections were confirmed by pyuria and urine culture positivity.

The main exposure variables were the number of total infections/pt-year and severe infections/pt-yr, categorized as below or above median, and number of subjects with and without infections or severe infections, all evaluated separately during induction and maintenance ISU. Predictors of infections, and infectious mortality were calculated.

Data were abstracted from the charts. Routine laboratory values, types of ANCA and antimyeloperoxidase (anti-MPO)/anti-proteinase 3 (anti-PR3) levels determined by indirect immunofluorescence and ELISA, and estimated GFR (eGFR), calculated with the CKD-EPI equation were tabulated. Birmingham Vasculitis Activity Score version 3 (BVAS) was calculated at the time of admission. Charlson comorbidity score (CCI) was assessed by determining conditions that had been present before AAV developed, and calculated without considering age and AAVcaused renal failure, as these variables were independently selected in multivariable analyses.

The study was approved by the local ethics committee (Institutional Review Board of St. Margit Hospital, No. 827/2012), and conducted in agreement with the Declaration of Helsinki. 
Statistical analysis was performed by using the IBM SPSS Statistics version 25.0 software package. Continuous variables were reported as mean \pm SD if normally distributed, and as median and interquartile range $[\mathrm{IQR}]$ if skewed. Comparisons between groups were analyzed by Student's $t$-tests for normally distributed, and Mann-Whitney U tests for non-parametric data. Predictors of infection were evaluated using logistic regression models and survival by Cox proportional hazard models, results are expressed as odds ratios (ORs) and hazard ratios (HRs) respectively, with 95\% confidence intervals (CIs) and $P$ values. For multivariable analyses those variables were selected that seemed important predictors according to univariable analyses or in clinical practice. Survival was calculated by Kaplan-Meier method, and log-rank tests to compare the groups. All tests were two-tailed and $P$ values of $<0.05$ were considered significant.

\section{RESULTS}

Between 1999 and 2019, 132 patients were diagnosed with AAV. Their age was $61.3 \pm 14$ years, $39 \%$ was male. Patients were categorized as PR3-ANCA (38\%) and MPO-ANCA positive (57\%) subjects; $5 \%$ of patients were ANCA negative. All participants had renal involvement, with baseline serum creatinine of 538 [IQR 444] $\mu \mathrm{mol} / \mathrm{L}, 67$ patients (51\%) needed hemodialysis (HD) at diagnosis. Pulmonary manifestations, nodules, masses with cavities or infiltrates were present in 57 patients (43\%). Based on the clinical presentation 40 of them (31\%) had pulmonary hemorrhage. ENT involvement was documented in 45 subjects (34\%). We found hypertension as the most frequent baseline comorbidity (in $65 \%$ of the patients), prevalence of diabetes was $8 \%$, coronary artery disease $8 \%$, cerebrovascular disease $8 \%$, COPD $12 \%$, congestive heart failure $4 \%$, liver cirrhosis $3 \%$, peripheral vascular disease $2 \%$, and malignant disease in the medical history in $8 \%$. Charlson comorbidity score, calculated without age and renal failure showed mean of $0.82 \pm 1.11$, median 0 [IQR 1], the BVAS was $21.1 \pm 5.6$. Baseline demographic data are summarized in Table 1.

IV bolus methylprednisolone was administered to 128 subjects in a dose of $11.4 \pm 3.9 \mathrm{mg} / \mathrm{kg}$, and given orally for everyone, initially $1 \mathrm{mg} / \mathrm{kg}$, and tapered gradually to the maintenance dose of $4 \mathrm{mg}$ daily or every other day in 5-6 months. IV bolus cyclophosphamide was given to 123 patients in $9.5 \pm 1.5 \mathrm{mg} / \mathrm{kg}$ dose, administered six times monthly, and repeated at months 9 and 12. Four subjects were treated with po CYC, and 5 subjects did not get this drug due to very old age and/or HD dependency and/or severe infections. During induction $43 \%$ of patients received prophylactic trimethoprim-sulfamethoxazole, at the discretion of the attending nephrologist. Long-term maintenance ISU therapy consisted of $4 \mathrm{mg}$ MP administered daily or every other day, and azathioprine given at the discretion of the attending nephrologist to 26 patients in a dose of $50-100 \mathrm{mg} /$ day.

During the total observation time 74 patients died (56\%), 5 patients were lost to follow-up and 11 were transplanted or transferred to another nephrological center. Infection was responsible for $32 \%$ of the total mortality; $21 \%$ of death occurred due to active vasculitis. Cardiovascular disease was responsible for death in $27 \%$, malignant disease in $7 \%$. In $13 \%$ of cases the cause of death remained undetermined. Dialysis could be discontinued in 34 subjects, but on long term further 11 patients progressed to ESRD. Fifty-one relapses occurred in 31 patients, with either rapid deterioration of earlier stabilized renal function or reactivation/new onset of pulmonary, gastrointestinal or cerebral manifestation of AAV. Gastrointestinal 
Table 1. Demographics, baseline data and comorbidities at time of diagnosis (mean \pm SD or median [interquartile range, IQR])

\begin{tabular}{|c|c|}
\hline Variable & Baseline data \\
\hline$n$ of patients & 132 \\
\hline Age (years) & $61.3 \pm 14$ \\
\hline Male/female $(n, \%)$ & $51 / 81(39 / 61 \%)$ \\
\hline Serum creatinine $(\mu \mathrm{mol} / \mathrm{L})$ & $538[444]$ \\
\hline Hemoglobin $(\mathrm{g} \%)$ & $8.6 \pm 1.5$ \\
\hline Serum albumin $(g / L)$ & $31.1 \pm 5.4$ \\
\hline $\mathrm{CRP}(\mathrm{mg} / \mathrm{L})$ & $44[89]$ \\
\hline Urinary protein excretion (mg/day) & $1,497[2,377]$ \\
\hline HD requirement on admission $(n, \%)$ & $67(51 \%)$ \\
\hline BVAS & $21.1 \pm 5.6$ \\
\hline $\begin{array}{l}\text { Charlson comorbidity score }{ }^{\mathrm{a}} \text { (mean } \pm \\
\mathrm{SD} / \text { median }[\mathrm{IQR}] \text { ) }\end{array}$ & $0.82 \pm 1.11 / 0[1]$ \\
\hline $\begin{array}{l}\text { Clinical diagnosis GPA/MPA/RLV/ } \\
\text { EGPA }(n, \%)\end{array}$ & $50 / 64 / 16 / 2(38 / 48 / 12 / 2 \%)$ \\
\hline $\begin{array}{l}\text { PR3-ANCA/MPO-ANCA positive/ } \\
\text { ANCA negative patients }(n, \%)\end{array}$ & $50 / 75 / 7(38 / 57 / 5 \%)$ \\
\hline $\begin{array}{l}\text { Anti-MPO level in MPO-ANCA } \\
\text { positive patients }\left(\mathrm{IU} / \mathrm{mL}^{\mathrm{b}}\right)\end{array}$ & $62 \pm 35$ \\
\hline $\begin{array}{l}\text { Anti-PR3 level in PR3-ANCA positive } \\
\text { patients }\left(\mathrm{IU} / \mathrm{mL}^{\mathrm{c}}\right)\end{array}$ & $81 \pm 29$ \\
\hline Dose of IV pulse MP ${ }^{\mathrm{d}}(\mathrm{mg} / \mathrm{kg}$ bw) & $11.4 \pm 3.9$ \\
\hline Dose of IV bolus $\mathrm{CYC}^{\mathrm{e}}(\mathrm{mg} / \mathrm{kg}$ bw) & $9.5 \pm 1.5$ \\
\hline Follow-up time (months) & 35 [85] \\
\hline
\end{tabular}

${ }^{\mathrm{a}}$ Charlson comorbidity score is calculated without age and renal function.

${ }^{b, c}$ Normal range for anti-MPO: 0-5 IU/mL, for anti-PR3: 0-8 IU/mL.

${ }^{\mathrm{d}}$ IV Methylprednisolone bolus was not given to 4 patients, they received only po corticosteroid.

${ }^{\mathrm{e}} \mathrm{IV}$ bolus cyclophosphamide was given to 123 patients; 4 patients received the drug orally; 5 patients were treated with corticosteroid and plasma exchange but without cyclophosphamide.

Abbreviations: $\mathrm{BVAS}=$ Birmingham vasculitis activity score; GPA $=$ granulomatosis with polyangiitis; MPA = microscopic polyangiitis; RLV = renal limited vasculitis; EGPA = eosinophilic granulomatosis with polyangiitis; $\mathrm{PR} 3=$ proteinase $3 ; \mathrm{MPO}=$ myeloperoxidase; $\mathrm{MP}=$ methylprednisolone; $\mathrm{CYC}=$ cyclophosphamide.

manifestation, presented with severe abdominal pain and bloody stool was proved as AAV complication by colonoscopy finding and histology data, and cerebral involvement presenting by confusion and cerebrovascular lesion by MRI studies. In those who did not need dialysis at the end of the observation, serum creatinine decreased to 175 [IQR 139] $\mu \mathrm{mol} / \mathrm{L}$ and eGFR improved to 31 [IQR 25] $\mathrm{ml} / \mathrm{min}$. Leucopenia developed in $9.3 \%$ and steroid diabetes in $14 \%$ of the participants.

During induction ISU 161 infections occurred; 76 of these were severe in 55 patients. During maintenance ISU, of the 113 infections 59 were severe in 30 patients. Moderately severe infections developed in 42 and 29 occasions during induction and maintenance ISU, respectively. The incidence of total and severe infections/pt-yr was $3.17 \pm 4.50$ (median 2 [IQR 4.21]) and 
Table 2. Baseline data of patient groups with total infections at rate below and above median during induction and maintenance immunosuppression

\begin{tabular}{|c|c|c|c|c|c|c|}
\hline \multirow[b]{2}{*}{ Variable } & \multicolumn{3}{|c|}{ During induction immunosuppression $(n=132)$} & \multicolumn{3}{|c|}{ During maintenance immunosuppression $(n=89)$} \\
\hline & $\begin{array}{l}\text { Below median }(<2 \\
\text { infections/pt-year })\end{array}$ & $\begin{array}{l}\text { Above median }(\geq 2 \\
\text { infections/pt-year) }\end{array}$ & $\begin{array}{c}P \\
\text { value }\end{array}$ & $\begin{array}{l}\text { Below median ( } 0 \\
\text { infections/pt-year) }\end{array}$ & $\begin{array}{l}\text { Above median }(>0 \\
\text { infections/pt-year })\end{array}$ & $\begin{array}{c}P \\
\text { value }\end{array}$ \\
\hline$n$ of patients & 58 & 74 & & 47 & 42 & \\
\hline Age (years) & $59.4 \pm 14$ & $62.6 \pm 13$ & 0.182 & $57.9 \pm 14$ & $60.6 \pm 13$ & 0.345 \\
\hline $\begin{array}{l}\text { Serum creatinine }(\mu \mathrm{mol} / \\
\mathrm{L})\end{array}$ & $400[478]$ & $606[410]$ & 0.037 & $426[410]$ & $538[558]$ & 0.437 \\
\hline Hemoglobin $(g \%)$ & $8.7 \pm 1.6$ & $8.5 \pm 1.4$ & 0.444 & $8.7 \pm 1.5$ & $8.7 \pm 1.7$ & 0.977 \\
\hline Serum albumin $(g / L)$ & $32.1 \pm 5.4$ & $30.3 \pm 5.2$ & 0.063 & $32.6 \pm 5.3$ & $31.2 \pm 5.5$ & 0.220 \\
\hline $\begin{array}{l}\text { HD requirement on } \\
\text { admission }(n, \%)\end{array}$ & $23(40 \%)$ & $44(60 \%)$ & 0.024 & $16(34 \%)$ & $22(52 \%)$ & 0.081 \\
\hline BVAS & $20.4 \pm 5.0$ & $21.6 \pm 6$ & 0.202 & $20.7 \pm 6.0$ & $20.1 \pm 5.1$ & 0.576 \\
\hline CCI & $0[1]$ & $1[2]$ & 0.001 & $0[0]$ & $1[1]$ & 0.001 \\
\hline $\begin{array}{l}\text { Clinical diagnosis GPA/ } \\
\text { MPA/RLV/EGPA (\%) }\end{array}$ & $41 / 47 / 12 / 0 \%$ & $35 / 50 / 12 / 3 \%$ & 0.576 & $47 / 42 / 11 / 0 \%$ & $38 / 50 / 10 / 2 \%$ & 0.613 \\
\hline $\begin{array}{l}\text { PR3-ANCA/MPO- } \\
\text { ANCA patients (\%) }\end{array}$ & $42 / 58 \%$ & $33 / 67 \%$ & 0.331 & $46 / 54 \%$ & $37 / 63 \%$ & 0.415 \\
\hline $\begin{array}{l}\text { Dose of IV pulse MP } \\
(\mathrm{mg} / \mathrm{kg} \mathrm{bw})\end{array}$ & $11.5 \pm 4.1$ & $11.3 \pm 3.8$ & 0.750 & $11.4 \pm 4.2$ & $11.2 \pm 3.7$ & 0.863 \\
\hline $\begin{array}{l}\text { Dose of IV bolus CYC } \\
(\mathrm{mg} / \mathrm{kg} \mathrm{bw})\end{array}$ & $9.6 \pm 1.5$ & $9.5 \pm 1.6$ & 0.838 & $9.1 \pm 1.3$ & $9.8 \pm 1.8$ & 0.077 \\
\hline Diabetes (\%) & $3 \%$ & $12 \%$ & 0.111 & $2 \%$ & $12 \%$ & 0.096 \\
\hline Leucopenia (\%) & $5 \%$ & $12 \%$ & 0.177 & $2 \%$ & $14 \%$ & 0.049 \\
\hline $\begin{array}{l}\text { Trimethoprim- } \\
\text { sulfamethoxazole } \\
\text { prophylaxis (\%) }\end{array}$ & $47 \%$ & $40 \%$ & 0.431 & $59 \%$ & $49 \%$ & 0.354 \\
\hline
\end{tabular}

Comparing gender, baseline CRP, anti-PR3, anti-MPO levels and urinary protein excretion between groups did not reveal statistically significant differences (data not shown).

Abbreviations: $\mathrm{BVAS}=$ Birmingham vasculitis activity score; GPA = granulomatosis with polyangiitis; MPA $=$ microscopic polyangiitis; RLV $=$ renal limited vasculitis; EGPA = eosinophilic granulomatosis with polyangiitis; CCI = Charlson comorbidity score, calculated without age and renal function; PR3 = proteinase 3; $\mathrm{MPO}=$ myeloperoxidase; $\mathrm{MP}=$ methylprednisolone; $\mathrm{CYC}=$ cyclophosphamide; pt-year $=$ patient-year

The bold values in table indicate significant levels of difference. 


\begin{tabular}{|c|c|c|c|c|c|c|}
\hline \multirow[b]{2}{*}{ Variable } & \multicolumn{3}{|c|}{ During induction immunosuppression $(n=132)$} & \multicolumn{3}{|c|}{ During maintenance immunosuppression $(n=89)$} \\
\hline & $\begin{array}{l}\text { Below median }(0 \\
\text { infections/pt-year) }\end{array}$ & $\begin{array}{l}\text { Above median }(>0 \\
\text { infections/pt-year) }\end{array}$ & $\begin{array}{c}P \\
\text { value }\end{array}$ & $\begin{array}{l}\text { Below median }(0 \\
\text { infection/pt-year })\end{array}$ & $\begin{array}{l}\text { Above median }(>0 \\
\text { infections/pt-year) }\end{array}$ & $\begin{array}{c}P \\
\text { value }\end{array}$ \\
\hline$n$ of patients & 77 & 55 & & 59 & 30 & \\
\hline Age (years) & $60.4 \pm 14$ & $62.2 \pm 13$ & 0.441 & $57.9 \pm 14$ & $61.7 \pm 11$ & 0.199 \\
\hline $\begin{array}{l}\text { Serum creatinine }(\mu \mathrm{mol} / \\
\mathrm{L})\end{array}$ & $434[463]$ & $640[402]$ & 0.002 & $369[458]$ & $604[543]$ & 0.057 \\
\hline Hemoglobin $(g \%)$ & $8.7 \pm 1.6$ & $8.5 \pm 1.4$ & 0.409 & $8.7 \pm 1.5$ & $8.7 \pm 1.7$ & 0.901 \\
\hline Serum albumin $(g / L)$ & $31.6 \pm 5.3$ & $30.4 \pm 5.4$ & 0.230 & $32.7 \pm 5.1$ & $30.6 \pm 5.7$ & 0.098 \\
\hline $\begin{array}{l}\text { HD requirement on } \\
\text { admission }(n, \%)\end{array}$ & $30(39 \%)$ & $37(67 \%)$ & 0.001 & $20(34 \%)$ & $18(60 \%)$ & 0.019 \\
\hline BVAS & $20.3 \pm 5.3$ & $22.1 \pm 5.9$ & 0.076 & $20.8 \pm 5.8$ & $19.7 \pm 5.2$ & 0.357 \\
\hline CCI & $0[1]$ & $1[2]$ & 0.086 & $0[1]$ & $1[1]$ & 0.001 \\
\hline $\begin{array}{l}\text { Clinical diagnosis GPA/ } \\
\text { MPA/RLV/EGPA (\%) }\end{array}$ & $35 / 51 / 12 / 2 \%$ & $42 / 45 / 13 / 0 \%$ & 0.568 & $46 / 44 / 10 / 0 \%$ & $37 / 50 / 10 / 3 \%$ & 0.474 \\
\hline $\begin{array}{l}\text { PR3-ANCA/MPO- } \\
\text { ANCA patients (\%) }\end{array}$ & $37 / 63 \%$ & $38 / 62 \%$ & 0.864 & $42 / 58 \%$ & $41 / 59 \%$ & 0.906 \\
\hline $\begin{array}{l}\text { Dose of IV pulse MP } \\
(\mathrm{mg} / \mathrm{kg} \mathrm{bw})\end{array}$ & $11.1 \pm 4.1$ & $11.8 \pm 3.6$ & 0.295 & $11.0 \pm 4.1$ & $12.0 \pm 3.7$ & 0.284 \\
\hline $\begin{array}{l}\text { Dose of IV bolus CYC } \\
(\mathrm{mg} / \mathrm{kg} \mathrm{bw})\end{array}$ & $9.6 \pm 1.7$ & $9.4 \pm 1.3$ & 0.417 & $9.1 \pm 1.4$ & $10.1 \pm 1.8$ & 0.015 \\
\hline Diabetes (\%) & $7 \%$ & $11 \%$ & 0.525 & $2 \%$ & $17 \%$ & 0.015 \\
\hline Leucopenia (\%) & $7 \%$ & $13 \%$ & 0.225 & $5 \%$ & $13 \%$ & 0.220 \\
\hline $\begin{array}{l}\text { Trimethoprim- } \\
\text { sulfamethoxazole } \\
\text { prophylaxis (\%) }\end{array}$ & $46 \%$ & $40 \%$ & 0.478 & $52 \%$ & $66 \%$ & 0.543 \\
\hline
\end{tabular}

Comparing gender, baseline CRP, anti-PR3 anti-MPO levels and urinary protein excretion between groups did not reveal statistically significant differences (data not shown).

Abbreviations: $\mathrm{BVAS}=$ Birmingham vasculitis activity score; $\mathrm{GPA}=$ granulomatosis with polyangiitis; $\mathrm{MPA}=$ microscopic polyangiitis; $\mathrm{RLV}=$ renal limited vasculitis; EGPA = eosinophilic granulomatosis with polyangiitis; $\mathrm{CCI}=$ Charlson comorbidity score, calculated without age and renal function; PR3 = proteinase 3; $\mathrm{MPO}=$ myeloperoxidase; $\mathrm{MP}=$ methylprednisolone; $\mathrm{CYC}=$ cyclophosphamide; pt-year $=$ patient-year.

The bold values in table indicate significant levels of difference. 
$1.51 \pm 2.50$ (median 0 [IQR 2]) during induction, and $0.35 \pm 0.66$ (median 0 [IQR 0.38]) and $0.19 \pm 0.43$ (median 0 [IQR 0.16]) during maintenance ISU, respectively.

Patients with lower than median incidence rate of total infections during induction $(<2 / \mathrm{pt}-$ yr) had lower baseline serum creatinine (400 [IQR 478] $\mu \mathrm{mol} / \mathrm{L}$ ), less dialysis requirement (40\%) and lower CCI (0 [IQR 1]), compared to those with $\geq 2$ infections/pt-yr (creatinine $606 \mu \mathrm{mol} / \mathrm{L}$ [IQR 410], $P=0.037$; dialysis requirement $60 \%, P=0.024$; CCI 1 [IQR 2] $P=0.001$; respectively). In remission, patient groups divided by the median number of infections/pt-yr (0 or $>0)$ showed significant difference in CCI ( 0 [IQR 0] versus 1 [IQR 1 ], $P=0.001)$ and in the occurrence of leucopenia at any time (2\% versus $14 \%, P=0.049)$, but not in baseline renal function, azathioprine administration or any other clinical data (Table 2).

Comparing patient groups below and above than median number of severe infections/pt-yr $(0$ or $>0)$ during induction, significant differences were found in baseline renal function (creatinine 434 [IQR 463] versus 640 [IQR 402] $\mu \mathrm{mol} / \mathrm{L}, P=0.002$, resp.) and dialysis requirement (39\% versus $67 \%, P=0.001$ resp.). During maintenance ISU, baseline dialysis requirement, diabetes, CCI and dose of bolus CYC administered as induction therapy differed significantly ( $34 \%$ versus $60 \%, P=0.019 ; 2 \%$ versus $17 \%, P=0.015 ; 0$ [IQR 1 ] versus 1 [IQR 1 ] $P=0.001 ; 9.1 \pm 1.4$ versus $10.1 \pm 1.8 \mathrm{mg} / \mathrm{kg}, P=0.015$, respectively (Table 3 ).

In univariate analysis severe infection was predicted by baseline serum creatinine (OR 1.002 [CI 1.000-1.003], $P=0.006$ ) and CCI (OR 1.395 [CI 1.011-1.925], $P=0.043$ ). Pulmonary manifestation approached the level of significance $(P=0.063)$, but neither ENT manifestation, nor ISU drug doses reached significance. In multivariate analysis, baseline serum creatinine and pulmonary manifestation proved to be independent predictors for severe infection, corrected for age, CCI and ENT manifestation (Table 4). Baseline HD requirement increased the OR of severe infection during induction by 2.875 [CI 1.342-6.161], $P=0.007$, corrected for the same parameters. We did not find any predictive clinical parameter for severe infection in remission.

Survival, analyzing the entire observation period showed association with developing severe infection during induction ISU in univariate analysis (HR 2.373 [CI 1.480-3.806] $P<0.001$ ), as did age (HR 1.054 [CI 1.030-1.078] $P<0.001$ ), baseline serum creatinine (HR 1.001 [CI 1.0001.002] $P=0.004$ ), and CCI (HR 1.791 [CI 1.478-2.171] $P<0.001)$. Those patients who had ENT manifestation had lower risk of mortality (HR 0.590 [CI $0.350-0.992$ ], $P=0.047$ ). Severe infection predicted all-cause mortality after correcting for age, baseline creatinine, CCI, ENT and pulmonary manifestation in multivariable Cox regression model (HR 1.998 [CI 1.214-

Table 4. Predictors of severe infection during induction immunosuppression in multivariate logistic regression analysis. Odds ratio (OR) and 95\% confidence interval (CI)

\begin{tabular}{lccr}
\hline & OR & $95 \%$ CI & $P$ value \\
\hline Baseline serum creatinine $(\mu \mathrm{mol} / \mathrm{L})$ & 1.002 & $1.001-1.003$ & $\mathbf{0 . 0 0 5}$ \\
Pulmonary manifestation & 2.153 & $1.017-4.560$ & $\mathbf{0 . 0 4 5}$ \\
ENT manifestation & 0.855 & $0.382-1.917$ & 0.704 \\
Age (years) & 1.008 & $0.978-1.038$ & 0.612 \\
CCI & 1.289 & $0.904-1.837$ & 0.161 \\
\hline
\end{tabular}

Abbreviations: ENT $=$ Ear-nose-throat; $\mathrm{CCI}=$ Charlson comorbidity score, calculated without age and renal function.

The bold values in table indicate significant levels of difference. 
3.287] $P=0.006)$. Kaplan-Meier analysis documented significantly longer survival of those who did not have any severe infection or any kind of infection during induction therapy, compared to those who suffered these complications (Fig. 1 panel a and b).

Among severe infections we documented blood stream infections mostly in those patients who needed acute dialysis line. Abscesses in different localizations as cerebral, pulmonary, subphrenical, or pancreatic, were also experienced. One patient died of disseminated herpes zoster infection. Endocarditis, meningitis were also diagnosed. Among the high number of lower urinary tract infections most did not cause any significant clinical complication. The number of
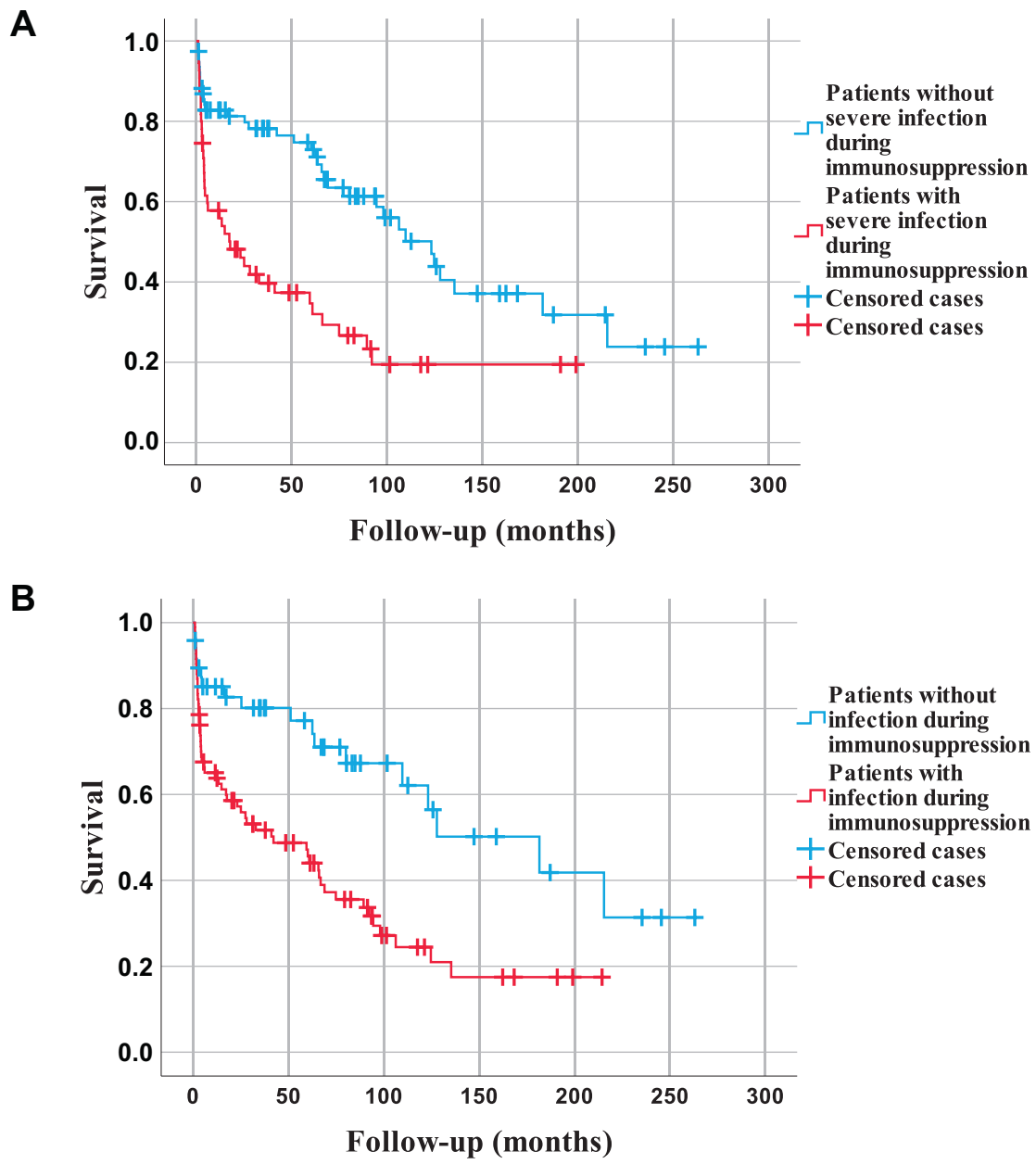

Fig. 1. Kaplan-Meier survival estimates during the entire observation period.

Legend to panel 1A.: Patients without severe infection during induction immunosuppression $(n=77)$ had significantly longer survival than those having such infection $(n=55)(P<0.001, \log$-rank test).

Legend to panel 1B.: Patients without any kind of infection during induction immunosuppression $(n=48)$ had significantly longer survival than those having infection $(n=84)(P=0.001$, log-rank-test $)$ 
Table 5. Types and localizations of infections with positive microbiological culture

\begin{tabular}{|c|c|c|c|c|c|c|c|}
\hline \multicolumn{2}{|c|}{ Causative organisms } & \multicolumn{6}{|c|}{ Localizations of the infections } \\
\hline & & $\begin{array}{c}\text { Blood } \\
\text { stream } \\
(n=36)\end{array}$ & $\begin{array}{c}\text { Respiratory } \\
\text { tract } \\
(n=25)\end{array}$ & $\begin{array}{c}\text { Urinary } \\
\text { tract } \\
(n=83)\end{array}$ & $\begin{array}{c}\text { GI } \\
\text { tract } \\
(n=8)\end{array}$ & $\begin{array}{c}\text { Skin and } \\
\text { joint } \\
(n=8)\end{array}$ & $\begin{array}{c}\text { CAPD } \\
\text { peritonitis } \\
(n=8)\end{array}$ \\
\hline \multirow[t]{7}{*}{ Bacterial } & Staph. aureus & 9 & 2 & 2 & & 2 & \\
\hline & Enterococcus & & & 9 & & & 1 \\
\hline & $\begin{array}{c}\text { Pseudomonas } \\
\text { aeruginosa }\end{array}$ & 3 & 7 & 3 & & & \\
\hline & Mycobacterium & & & & & 1 & \\
\hline & $\begin{array}{l}\text { Clostridioides } \\
\text { difficile }\end{array}$ & & & & 6 & & \\
\hline & $\begin{array}{l}\text { Other Gram } \\
\text { positives }\end{array}$ & 15 & & 1 & & & 6 \\
\hline & $\begin{array}{l}\text { Other Gram } \\
\text { negatives }\end{array}$ & 9 & 11 & 67 & 1 & 2 & 1 \\
\hline \multirow[t]{2}{*}{ Viral } & Herpes zoster & & & & & 3 & \\
\hline & CMV & & 1 & & & & \\
\hline Fungal & & & 4 & 1 & 1 & & \\
\hline
\end{tabular}

positive cultures was 168. Gram-negative bacteria were responsible for blood stream infections in $33 \%$, and for respiratory tract infections in $72 \%$ of cases. Urinary tract infections were caused by Gram-negative bacteria in 70 cases (84\%), the most prevalent species were E. coli in 36 and Klebsiella in 14 episodes. Types and localizations of the infections with positive microbiological cultures are summarized in Table 5.

\section{DISCUSSION}

In our cohort of patients with severe systemic AAV we found a high number of infections, with almost 10 times more frequent occurrence during induction than maintenance ISU. Both severe infections and any kind of infections showed association with baseline renal function. Advanced kidney failure, especially if dialysis was required at diagnosis, multiplied the risk for infectious complications. Those patients who presented with comorbidities also faced higher risk of such complications. Seventy-two percent of respiratory tract infections were caused by Gram-negative bacteria, and blood stream infections were also caused by these types of microorganisms in onethird of the cases. Subjects with severe or any kind of infection had significantly higher all-cause mortality than those without such complication.

According to published clinical reports infections are the most frequent complication of ISU therapy, occurring in $20-76 \%$ of the patients with AAV, depending on the length of observation and the severity of the clinical condition $[7,8,10,12-17]$. We found very high incidence rate of infections, which we explain by the fact that patients were recruited at a nephrology center with severe renal failure - more than half of them needed dialysis at diagnosis -, and also by multiorgan involvement, shown by high BVAS. We documented all infectious episodes including mild low urinary tract infections, which latter were extremely prevalent in our cohort even 
during remission. The clinical significance of low urinary tract infection is questionable, but may indicate insufficient anti-inflammatory resistance in the urinary tract as a long-term consequence of the condition. Although most of the severe infections occurred during induction therapy, these also developed in remission when low dose maintenance ISU was administered, indicating that AAV patients in this stage still have high risk of such complications.

Several investigators documented the respiratory tract as the site of the highest incidence of infections, beside urinary tract and the blood stream $[19,12,7,14,16]$. Damage of the local protective barrier in lungs likely enhances the risk of such complication [17]. Our finding that pulmonary involvement is a significant predictor of severe infection is in accordance with this observation: those patients who had either lung hemorrhage or other pulmonary manifestation as nodules or masses with cavities had higher incidence of respiratory tract infection. Interestingly, our results showed Gram-negative bacterial predominance as causative agent in respiratory tract infections, which is in agreement with the findings of Yang and coworkers in Chinese AAV patients [17]. This indicates that the initial, "ex juvantibus" antibiotic treatment should be effective to such bacteria as well. With blood stream infections Gram-negative antibiotic coverage is also important, but especially in patients with central venous dialysis catheter Staphylococcus aureus is still the most prevalent microbiological organism [16].

Many, but not all previous studies showed that baseline kidney function was associated with infections $[1,7,12,14,15,17,18]$. The discrepancy can likely be explained by different stages of renal failure in these cohorts. In our group of patients with advanced renal failure, those with worse baseline renal function experienced significantly higher number of severe and also total number of infections during induction ISU, and even in remission if dialysis was necessary at baseline. We assume that the uremic condition itself increases the risk to such complications, beside the side effect of ISU.

All-cause mortality showed associations both with severe and total number of infections in our analysis. Infections were responsible for $32 \%$ of mortality. Most of the previous investigations presented similar data, underlining the importance of infection prevention aiming to reduce mortality $[7,13,14,19]$. Interestingly, patients who presented with ENT involvement did not develop severe infections more frequently than subjects without it, and, in accordance with previous findings even showed more favorable long-term survival [13]. ISU, with high enough drug doses and sufficient length of administration is a key element of AAV treatment to ensure improved survival, but at the same time promotes infection [8, 9, 20]. Among previously published clinical investigations several documented association between infections and the dose of CYC $[9,17]$, whereas others found high dose and prolonged administration of corticosteroids responsible for such complication $[18,21,22]$. On the other hand, when shorter course of ISU is administered, the number of infections may decrease but relapse may develop more frequently, which necessitates repeating high dose induction ISU [23].

We did not find such association with corticosteroid dose, likely because of the highly protocolized therapy with very little variations in the dosages. In the subgroup with higher than median number of severe infections/pt-yr during maintenance ISU the induction CYC dose was higher, and the total number of infections/pt-yr was also numerically higher, although this latter did not reach statistical significance. We may speculate that those who received a higher dose of CYC during induction remained more immune-compromised on long term.

It is well documented that if CYC causes leucopenia, severe infectious episodes develop more frequently [19]. Our data also refer to this finding, although statistically we could not prove it. 
The preventive role of trimethoprim-sulfamethoxazole also cannot be questioned $[3,5,9,13$, 20], and the reason why our data did not show its usefulness is likely because no Pneumocystis jiroveci infection was detected in our patients.

Some investigators found increased incidence of infections in elderly patients on ISU therapy $[14,15]$. In our analysis age did not differ significantly between the subgroups with lower and higher than median infections/pt-year. According to our experience elderly patients with AAV can be successfully treated with appropriately dosed immunosuppressive medications, as we and others documented in previous clinical studies [24, 25].

The novelty of our investigation is the significant role of baseline comorbidities as independent risk factors for infections. It has already been documented that poor baseline functional status increases the risk of mortality in AAV [26]. According to our present analysis, in patients who develop AAV with other diseases in their medical history need even more attention toward infections compared to those in whom AAV is the single abnormality.

The finding that renal function is one of the basic determinants of infectious complications' risk refers to the well documented fact that early diagnosis is crucial to decrease morbidity and mortality in AAV [4]. The attending team's experience with the disease also has to be emphasized, as it has an important role in education of both patients and medical staff, and in the reinforcement of preventive measures including personal hygiene, prophylactic vaccinations, and also the prompt recognition and treatment of the infections. This view is supported by our finding that less infections developed and the patient survival was longer in the second decade of our 20-year observation, even after correcting for age, renal function, CCI and dose of immunosuppressive drugs (data not shown).

Our study has some limitations. First, it is a retrospective data analysis. Yet, we believe that careful data collection supported reliable results. Second, this study was conducted in a tertiary nephrological center, with exceptionally severe disease manifestations, represented by high dialysis requirement and multiorgan manifestations requiring intensive ISU therapy, which cannot apply to all patients diagnosed with AAV but less severe renal failure or organ involvement. We decided to include episodes of CAPD peritonitis and Clostridioides difficile infections in our analysis, as the incidence of these complications was likely increased by immunosuppression. Furthermore, the severity of the infections was categorized arbitrarily, which may substantially influence the findings. Yet, we think that the categories we applied in our investigation followed appropriately their clinical relevance.

\section{CONCLUSION}

Advanced renal failure, pulmonary involvement and high degree of comorbidities increase the risk of infectious complications along the course of AAV. Those who suffer infection during induction immunosuppression have significantly poorer long-term survival. The infections are often severe and develop in atypical localizations, for which the attending physician has to be prepared. Our findings indicate the need for high vigilance for infections, and also refer to the obligation of close follow-up of the comorbid conditions when treating patients with AAV. These measurements, which likely improve outcome, have to be evaluated in future prospective clinical investigations. 


\section{ACKNOWLEDGMENTS}

The authors are indebted to all colleagues who participated in the care of the patients studied at the Nephrology Department of St. Margit Hospital between 1999 and 2019. We are thankful for Magdolna Kardos and Attila Fintha, nephropathologists at the Semmelweis University, for analyzing renal biopsy specimens since 2016, and to József Budai, specialist of infectious diseases, Dél-Pesti Centrum Hospital, for advising clinical management of patients with infections.

\section{REFERENCES}

1. File I, Pucsok K, Trinn Cs, Ujhelyi L, Balla J, Mátyus J. Clinical consequences and significance of antineutrophil cytoplasmic antibody positivity in anti-glomerular membrane disease. (in Hungarian) Orv Hetil 2013; 154: 1696-701.

2. Hirayama K, Kobayashi M, Usui J, Arimura Y, Sugiyama H, Nitta K, et al. Pulmonary involvements of antineutrophil cytoplasmic autoantibody-associated renal vasculitis in Japan. Nephrol Dial Transpl 2015; 30: i83-93.

3. Szabó M, Pálfi P, Bazsó A, Poór Gy, Kiss E. Recent advances in the treatment of antineutrophil cytoplasm antibody associated vasculitides. (in Hungarian) Orv Hetil 2015; 156: 1653-60.

4. Westman K, Flossmann O, Gregorini G. The long term outcomes of systemic vasculitis. Nephrol Dial Transpl 2015; 30: i60-6.

5. Yamagata K, Usui J, Saito C, Yamaguchi N, Hirayama K, Mase K, et al. ANCA-associated systemic vasculitis in Japan: clinical features and prognostic changes. Clin Exp Nephrol 2012; 16: 580-8.

6. Miloslavsky EM, Specks U, Merkel PA, Seo P, Spiera R, Langford CA, et al. Clinical outcomes of remission induction therapy for severe antineutrophil cytoplasmic antibody-associated vasculitis. Arthritis Rheum 2013; 65: 2441-9.

7. Kitagawa K, Furuichi K, Sagara A, Shinozaki Y, Kitajima S, Toyama T, et al. Risk factors associated with relapse or infectious complications in Japanese patients with microscopic polyangiitis. Clin Exp Nephrol 2016; 20: 703-11.

8. Little MA, Nightingale P, Verburgh CA, Hauser T, De Groot K, Savage C, et al. Early mortality in systemic vasculitis: relative contribution of adverse events and active vasculitis. Ann Rheum Dis 2010; 69: 1036-43.

9. Turnbull J, Harper L. Adverse effects of therapy for ANCA-associated vasculitis. Best Pract Res Clin Rheum 2009; 23: 391-401.

10. Flossmann O, Berden A, de Groot K, Hagen C, Harper L, Heijl C, et al. Long-term patient survival in ANCAassociated vasculitis. Ann Rheum Dis 2011; 70: 488-94.

11. Jennette JC, Falk RJ, Bacon PA, Basu N, Cid MC, Ferrario F, et al. 2012 Revisited international Chapel Hill Consensus conference nomenclature of vasculitides. Arthritis Rheum 2013; 65: 1-11.

12. Charlier C, Henegar C, Launay O, Pagnoux C, Berezné A, Bienvenu B, et al. Risk factors for major infections in Wegener granulomatosis: analysis of 113 patients. Ann Rheum Dis 2009; 68: 658-63.

13. Kronbichler A, Jayne DRW, Mayer G. Frequency, risk factors and prophylaxis of infection in ANCA-associated vasculitis. Eur J Clin Invest 2015; 45: 346-68.

14. Lai QY, Ma TT, Li ZY, Chang DY, Zhao MH, Chen M. Predictors of mortality in patients with antineutrophil cytoplasmic autoantibody-associated vasculitis: a study of 398 Chinese patients. J Rheumatol 2014; 41: 1849-55. 
15. McGregor JG, Negrete-Lopez R, Poulton CJ, Kidd JM, Katsanos SL, Goetz L, et al. Adverse events and infectious burden, microbes and temporal outline from immunosuppressive therapy in antineutrophil cytoplasmic antibody-associated vasculitis with native renal function. Nephrol Dial Transpl 2015; 30: i171-81.

16. Weidanz F, Day CJ, Hewins P, Savage CO, Harper L. Recurrences and infections during continuous immunosuppressive therapy after beginning dialysis in ANCA-associated vasculitis. Am J Kidney Dis 2007; 50: 36-46.

17. Yang L, Xie H, Liu Z, Chen Y, Wang J, Zhang H, et al. Risk factors for infectious complications of ANCAassociated vasculitis: a cohort study. BMC Nephrol 2018; 19: 138.

18. Goupil R, Brachemi S, Nadeau-Fredette AC, Déziel C, Troyanov Y, Lavergne V, et al. Lymphopenia and treatment-related infectious complications in ANCA-associated vasculitis. Clin J Am Soc Nephrol 2013; 8: 416-23.

19. Booth AD, Almond MK, Burns A, Ellis P, Gaskin G, Neild GH, et al. Outcome of ANCA-associated renal vasculitis: a 5-year retrospective study. Am J Kidney Dis 2003; 41: 776-84.

20. Wong L, Harper L, Little MA. Getting the balance right: adverse events of therapy in anti-neutrophil cytoplasm antibody vasculitis. Nephrol Dial Transpl 2015; 30: i164-70.

21. McGregor JG, Hogan SL, Hu Y, Jennette CE, Falk RJ, Nachman PH. Glucocorticoids and relapse and infection rates in anti-neutrophil cytoplasmic antibody disease. Clin J Am Soc Nephrol 2012; 7: $240-7$.

22. Watanabe-Imai K, Harigai M, Sada KE, Yamamura M, Fujii T, Dobashi H, et al. Clinical characteristics of and risk factors for serious infection in Japanese patients within six months of remission induction therapy for antineutrophil cytoplasmic antibody-associated vasculitis registered in a nationwide, prospective inception cohort study. Mod Rheumatol 2017; 27: 646-51.

23. de Joode AAE, Sanders JSF, Rutgers A, Stegeman CA. Maintenance therapy in antineutrophil cytoplasmic antibody-associated vasculitis: who needs what and for how long? Nephrol Dial Transpl 2015; 30: i150-8.

24. Haris Á, Polner K, Arányi J, Braunitzer H, Kaszás I, Mucsi I. Clinical outcomes of ANCA-associated vasculitis in elderly patients. Int Urol Nephrol 2014; 46: 1595-600.

25. Weiner M, Goh SM, Mohammad AJ, Hruskova Z, Tanna A, Bruchfeld A, et al. Outcome and treatment of elderly patients with ANCA-associated vasculitis. Clin J Am Soc Nephrol 2015; 10: 1128-35.

26. Little MA, Nazar L, Farrington K. Outcome in glomerulonephritis due to systemic small vessel vasculitis: effect of functional status and non-vasculitic co-morbidity. Nephrol Dial Transpl 2004; 19: 356-64.

Open Access. This is an open-access article distributed under the terms of the Creative Commons Attribution 4.0 International License (https://creativecommons.org/licenses/by/4.0/), which permits unrestricted use, distribution, and reproduction in any medium, provided the original author and source are credited, a link to the CC License is provided, and changes - if any - are indicated. (SID_1) 